\title{
Anaphylaxis after horsefly sting: a strange case of wasp-horsefly syndrome
}

\author{
Alessandro Buonomo, Angela Rizzi, Arianna Aruanno, Eleonora Nucera \\ UOSD Allergologia, Fondazione Policlinico Universitario Agostino Gemelli IRCCS, Rome, Italy \\ Adv Dermatol Allergol 2021; XXXVIII (2): 331-332 \\ DOI: https://doi.org/10.5114/ada.2021.106212
}

Horseflies belong to the Diptera order and encompass several families such as Tabanidae. They are haematophagous insects that can also bite humans. Their bite is painful and may provoke a local wheal and flare reaction. Systemic reactions till anaphylaxis are also described in the literature [1-3].

The so-called wasp-mosquito syndrome has already been investigated, and hyaluronidase was shown to be the cross-reactive allergen between Hymenoptera venom and mosquito saliva $[4,5]$.

We describe the case of a 45-year-old man who experienced an anaphylactic reaction (general malaise, unconsciousness, and loss of sphincteral control) immediately after 3 horsefly bites on the trunk. He was immediately treated with intramuscular epinephrine $0.5 \mathrm{mg}$ and betamethasone $8 \mathrm{mg}$ by emergency medical personnel and then transported to the emergency department of our hospital. Here he underwent a head computed tomography (CT) scan with a negative result and was then discharged in good clinical conditions. Serum tryptase was not measured after the acute event.

After 2 months, the patient was referred to our allergy unit. He underwent intradermal tests with Apis mellifera (Aquagen, ALK-Abelló), Vespula spp. (Aquagen, ALKAbelló), and Polistes dominula (Pharmalgen, ALK-Abelló) venoms. Basal tryptase as well as total and specific lgE to whole venoms (horsefly, Polistes dominula, Vespula spp., Vespa crabro) and to molecular components (Ves $\vee 5$, Pol d 5, Ves v 1) were assessed (Thermo Fisher Scientific, Waltham, USA).

Intradermal tests were positive for Vespula and Polistes venoms at a concentration of $100 \mathrm{USQ} / \mathrm{ml}$ and $0.01 \mu \mathrm{g} / \mathrm{ml}$, respectively.

Basal tryptase was within the normal range $(9.3 \mu \mathrm{g} / \mathrm{l})$ while specific IgEs were positive for all the venoms tested (Table 1).
The patient was then provided an emergency action plan, including auto-injectable epinephrine. Specific immunotherapy with wasp venom was not taken in consideration because the patient had been stung by wasps several times with no systemic reactions. We also took in consideration the possibility of a systemic mastocytosis (REMA score $=+3$ ), but the patient refused to undergo a bone marrow biopsy.

Horseflies are hematophagous biting insects. Their saliva contains a wide range of molecules active on blood and immune systems, but the relevant allergens are not well characterized.

In the literature several reports describe systemic reactions to Diptera bites in patients with a clinical history of systemic reactions to Hymenoptera stings.

Some authors showed the relevant allergen of horsefly is a $69 \mathrm{kD}$ salivary gland protein that can bind IgE. These authors also stated that this protein could not cross-react with relevant Hymenoptera allergens because of its higher molecular weight [1].

Other authors identified and characterized 2 IgEbinding proteins from the salivary glands of the horsefly Tabanus yao, called Tab y 1 and Tab y 2, with molecular weight of 26 and $35 \mathrm{kDa}$, respectively. Tab y 1 is an Ag 5 -like protein that is the major allergen of Vespidae, and Tab y 2 is a hyaluronidase with a $60 \%$ sequence similarity to Vespid hyaluronidase (Ves $\vee 2)$. These findings provide support for the presence of the wasp-horsefly syndrome [6].

Tab y 1, a 70-kDa apyrase, was identified as a new major horsefly allergen [7]. This protein is able to inhibit platelet aggregation and have antithrombotic activity. Tab y 1 may also induce basophil activation. Tab y 1 does not cross-react with other Hymenoptera venoms, which might explain the primary sensitizations to Diptera bites as previously reported [1].

Address for correspondence: Alessandro Buonomo, UOSD Allergologia, Fondazione Policlinico Universitario Agostino Gemelli IRCCS, Largo Gemelli 8, 00168 Rome, Italy, phone: +393476650135, e-mail: alessandro.buonomo@policlinicogemelli.it Received: 3.07.2019, accepted: 25.07.2019. 
Table 1. Laboratory test results

\begin{tabular}{lc}
\hline Allergen & Result [U/ml] \\
\hline Horsefly, i204 & 4.17 \\
\hline Vespula spp. (yellow jacket), i3 & 9.87 \\
\hline Vespa crabro, i75 & 6.17 \\
\hline Polistes dominula (paper wasp), i77 & 80.5 \\
\hline Ves v 1, i211 & 0.18 \\
\hline Ves v 5, i209 & 7.41 \\
\hline Pol d 5, i77 & 50.0 \\
\hline
\end{tabular}

Our patient showed a primary sensitization to Hymenoptera venoms (Table 1) with a clinical history of local reactions, and so we think cross-reactive allergens might be involved.

No specific treatment is available for these patients, and specific immunotherapy with Vespid venoms seem not to be effective. In fact, some patients with systemic reactions to Diptera bites were under immunotherapy with Vespid venoms and the treatment was not protective. Moreover, we must take in consideration that Tab y 1 sensitization may also occur, and this is a unique Diptera allergen [2, 3].

So, in the case of systemic reactions to Diptera bites, the patient should be provided with an emergency action plan and trained to use an epinephrine auto-injector.

Further studies are needed to identify other relevant allergens of Diptera saliva and to improve the diagnostic tools.

\section{Conflict of interest}

The authors declare no conflict of interest.

\section{References}

1. Hemmer W, Focke M, Vieluf D, et al. Anaphylaxis by horsefly bites: identification of a $69 \mathrm{kd}$ lgE-binding salivary gland protein from Chrisops spp. (Diptera Tabanidae) by western blot analysis. J Allergy Clin Immunol 1998; 101: 134-6.

2. Quercia O, Emiliani F, Foschi FG, et al. The wasp-horsefly syndrome. Eur Ann Allergy Clin Immunol 2008; 40: 61-3.

3. Quercia O, Emiliani F, Foschi FG, et al. A case of anaphylaxis: horse-fly or hymenoptera sting? Eur Ann Allergy Clin Immunol 2009; 41: 152-4.

4. Sabbah A, Hassoum S, Drouet M, et al. The wasp-mosquito syndrome. Allerg Immunol (Paris) 1999; 31: 175-84.

5. Sabbah A, Hassoum S, Drouet M, et al. The wasp-mosquito syndrome: extension of cross-allergenicity to the horsefly. Allerg Immunol 2000; 32: 16-9.

6. Ma D, Li Y, Dong S, et al. Purification and characterization of two new allergens from the salivary glands of the horsefly, Tabanus yao. Allergy 2011; 66: 101-9.

7. An S, Ma D, Wei JF, et al. A novel allergen Tab y 1 with inhibitory activity of platelet aggregation from salivary glands of horseflies. Allergy 2011; 66: 1420-7. 